\title{
Analysis of multichannel optical rotary connectors based on the compensation operating principle with mirror and prismatic optical compensators (Part 1)
}

\author{
V.M. Shapar ${ }^{1}$, V.S. Lysenko ${ }^{1}$, A.V. Savchuk ${ }^{2}$ \\ ${ }^{I} V$. Lashkaryov Institute of Semiconductor Physics, NAS of Ukraine, \\ 41, prospect Nauky,03028 Kyiv,Ukraine,e-mail:v_shapar@rambler.ru \\ ${ }^{2}$ International center "Institute for Applied Optics", NAS of Ukraine, \\ 10-g, Kudryavska str., 04053 Kyiv, Ukraine
}

\begin{abstract}
Performed in this work is a comprehensive theoretical computer analysis of performances inherent to two types of multichannel optical rotary connectors (ORC) of compensation operation based on mirror and prismatic compensators. This analysis relies on exact analytical expressions obtained for light ray paths in ORC models with a mirror compensator made in the form of bilateral mirror placed between two optical hemispheres and with prismatic compensator made in the form of Dove prism placed between two non-aberrational elliptic lenses.

Found in ORC with the mirror compensator is the essential deficiency inherent to all these constructions, which is related with considerable rotary oscillations in the value of optical signals in mirror angular positions when the mirror halves the input light beam. In these mirror positions, the amplitude value of optical signal oscillations exceeds $95 \%$, and optical losses are higher than $-13 \mathrm{~dB}$, when the rotor turns. One deficiency more in these constructions is also strict technical requirements to the accuracy of making the optical components and mechanisms at the level of $1 \ldots 2 \mu \mathrm{m}$.

Concerning the ORC construction with a prismatic compensator as well as collimator and focusing lenses common for all the channels, one should note the inadmissibly high optical losses of the signal value (higher than $-30 \ldots 40 \mathrm{~dB}$ ) in the case of construction with fiber-optic interfaces, and large dimensions and mass in the case of active construction with optoelectronic transducers at the inputs and outputs of ORC. For example, when the number of channels $N=10$ the longitudinal dimension of optical transfer channel (prism and lenses) exceeds $300 \mathrm{~mm}$, and this dimension increases with increasing the number of channels. When this dimension is lower than $100 \mathrm{~mm}$, the facility can be equipped with only one optical communication channel containing one LED and one photodiode located on the rotation axis. Optical losses in these constructions cannot be also considered as the satisfactory ones, since the respective loss value is higher than $-18 \mathrm{~dB}$ for the number of channels $N=10$.
\end{abstract}

Keywords: optical rotary connector, fiber-optic rotary connector, fiber-optic communication, optical compensator, optoelectronic.

Manuscript received 12.09.16; revised version received 25.01.17; accepted for publication 01.03.17; published online 05.04.17.

\section{Introduction}

To transfer information signals from rotating objects to the stationary ones in various branches of science and technique, different contact, capacitive and inductive current-collecting connectors (e.g., of the "Slip ring" type) are widely used up to date. The contact connectors are the most often applied, since they are more simple and cheap.

Also, with development of information technologies and impetuous growth of information volumes that 
should be transferred from rotating objects to the stable ones, implementation of fiber-optic transducers for physical quantities into rotating objects as well as fiberoptic transmission lines, traditional application of contact current collectors in many cases of up-to-date technique is not only problematic but sometimes impossible in general because of principal physical deficiencies inherent to current-collecting facilities. Among these deficiencies, it should be noted first of all the limited frequency band of electric transmission channels based on contact rings, high sensitivity to external electromagnetic interference as well as great mass and considerable sizes of these facilities.

A low frequency band of electric transmission channels and sizeable mass/dimension characteristics (e.g., of Sleep rings) in the multichannel version cause especial difficulties when using them in the objects intended for transferring huge information signal massifs. For instance, the mass of information parts of contact current collectors used in observational radars is approximately $200-300 \mathrm{~kg}$, and their dimensions reach several meters in length and $0.5 \mathrm{~m}$ in diameter [1].

In up-to-date radar complexes with phased-array antennas, which are characterized by considerably higher information fluxes that should be transferred from radio-electronic facilities mounted on the rotating antenna-spinning column of the complex to stationary modules, the amount of rotary current collectors must reach several thousands units instead of hundred ones, as earlier. It will result in further complication of current collectors that are already over-saturated by the amount of electrical channels, without this addition. In this case, the mass of cable assemblies intended for transferring all the sets of electric signals between radio-electronic modules operating in the observational radar will reach more than ten tons.

The same difficulties arise when using the currentcollecting facilities in modern systems for reconnaissance, surveillance and target acquisition as well as for vehicle navigation technologies [2].

Optimal solution of the problem of transferring huge massifs of high-frequency wide-band signals from rotating objects to the stationary ones is now possible by using, instead of the above current-collecting facilities, their functional contactless analogs - optic rotary connectors (ORC), and applying optical communication channels for this process of information transfer.

Optic rotary connectors are delivered from the above deficiencies of current collectors and possess a set of unique properties and advantages over the previous ones.

Their main advantages are as follows:

- $\quad$ absence of frictional contacts;

- $\quad$ wide frequency band of the transmission channel, which exceeds the frequency band of electric channels by thousand times;

- mass and dimensions of ORC are hundreds times lower than those of their electric analogs.

\section{Besides, ORC}

- provide galvanic separation between input and output of the communication channel in ORC, which enables to connect radio-electronic units that are under electric potentials with the difference of tens or hundreds kilovolts;

- allow transferring the optical signals within the frequency range from direct current up to superhigh frequencies even simultaneously in both directions;

- $\quad$ are absolutely insensitive to actions of external electromagnetic interference;

- do not create electric reactivity in communication channels;

- $\quad$ guarantee safety of their application at the objects operating in dangerous explosive conditions, which has its great social implication.

The above mentioned advantages of ORC as well as their high reliability and longevity, absence of any needs in preventive maintenance for 3 to 5 years correspond to requirements of up-to-date technique, and owing to it ORC can be undoubtedly related to one of the most promising facilities for signaling between movable and stationary objects and find their more and more wide use in various branches of surface and space technique.

Unfortunately, up to now considerable achievements are only reached in development of single- and two-channel ORC [3-6]. The frequency band of the optical channel in them comprises the range $0.1-10 \mathrm{Gbit} / \mathrm{s}$, and in some facilities can even exceed the latter value. The channel can be bi-directional and full-duplex, i.e., it can transfer information in both directions simultaneously. For this case, they use the technique of using two different wavelengths for transferred signals (most often they are $\lambda=1550 \mathrm{~nm}$ in one direction and $\lambda=$ $1310 \mathrm{~nm}$ in the opposite one) [2].

Single-channel facilities can be also used in the multichannel regime. In this case, they use the technique of wavelength division multiplexing (WDM) [2, 7-10]. However, there is a considerable amount of cautions when multiplexing is not desirable. Namely: when one should provide secrecy in channels operating in parallel with channels transmitting unclassified information; when there is a necessity to have an additional cashed circuit aimed at providing high reliability of data transfer; to avoid cross-interference between channels; when the data are received from different places; when it is necessary to have a free space at the device axis to provide allocation of additional facility inside ORC, e.g., SHF-waveguide, etc [2, 11].

Therefore, many researches in the leading countries all over the world perform intense investigations for creation of multichannel ORC with parallel independent physical channels. They study possibilities for using various physical principles and optical elements aimed at creating ORC.

Beside traditional geometrical optics, there studied are the possibilities to apply holographic, Fresnel, fiber 
and gradient optics, as well as electro- and magnetooptics. In these cases, they use various optical facilities and elements, namely: ring fiber-optic transducers of the light beam shape [12-20], holographic Fresnel lenses [21-25], focusing facilities [26, 27], light-guide facilities based, in particular, on optical fibers and hollow mirror toroids [28-32], mirror systems that revolve synchronically $[2,33,34]$, etc. As a separate direction, one should name development of facilities based on the principle of compensation [35-50]. From the viewpoint of reaching the maximum possible number of optical channels, the latter are the most promising ones up to date.

ORC based on the compensation principle of action essentially differs, from the construction viewpoint, from other ORC types in that the latter contain an additional slide assembly for optical interconnection, the rotation velocity of which differs from that of the ORC rotor part. On the one hand, availability of this additional movable compensation assembly is a deficiency of these devices, since complicates the facility, but on the other hand, the very compensation principle enables to create ORC designed for tens of optical channels as opposite to the other ORC types where the amount of optical channels does not exceed 3 to 8 channels, in practice.

Optical-and-technical characteristics of ORC with a compensator mainly depend on properties of the compensator used in it. Known up to date is a set of technical solutions for ORC with prismatic [35-43], reflective [44-47] and fiber-optic compensators [48-51]. A considerable amount of these technical solutions are studied neither experimentally nor theoretically and need a deep theoretical analysis.

Shown in this analytical review are the results of computer simulation only part of the known technical solutions ORC based on the compensation principle realized with a mirror compensator and that with the Dove prism, including the focusing and collimating lenses that serve as the collective ones in all the channels.

\section{Multichannel ORC with prismatic compensators}

One of the first constructions of ORC with prismatic compensator was offered yet in 1977 by Myren L. Iverson who used the Dove prism [35]. This facility should be related to active constructions [52], inputs and out puts of which are provided with respective optoelectronic transducers for conversion of the electric signal into the optical one in the transmitting part of the communication channel and, vice versa, of the optical signal into the electric one in the receiving part (for example, LEDs in the transmitting part and photodetectors in the receiving part). Active facilities do not operate in the composition of fiber-optic transmission lines but only in the composition of electric lines. Therefore, their advantages over other collectors lies only in the fact that they are less sensitive to external electromagnetic interferences and are more reliable.
The essential feature of the Iverson construction is that for focusing and collimating the light beams there used are focusing and collimating collective lenses for all the transmission channels. As it will be shown below, the principle for image formation at the output ORC interface by using the collective lens has essential deficiencies, namely: the amount of optical channels in the active design with optoelectronic transducers is limited by only a few channels, because of a high level of the cross-interference between them and, in the case of passive variant used for facility composition with optical interfaces designed for operation directly in the composition of fiber-optic transmission lines, very rigorous requirements to the facility components and their assemblage.

Also, there exists a number of technical solutions for ORC with prismatic compensators, where individual focusing and collimating lenses are used in each optical channel [36-43]. These constructions possess better technical performances as compared with those mentioned above, in particular, the amount of optical channels can be increased here. Since the mentioned constructions have structural peculiarities that essentially influence on their technical performances, it seems reasonable to consider them as typical representatives of two different directions for creation of multichannel ORC with prismatic compensators. Analysis of these constructions will be presented in the following publication.

\subsection{Multichannel ORC with prismatic compensators and lenses for focusing and collimation that serve as the collective ones in all the channels}

Let us consider this ORC type by using, as an example, the construction [35] schematically depicted in Fig. 1.

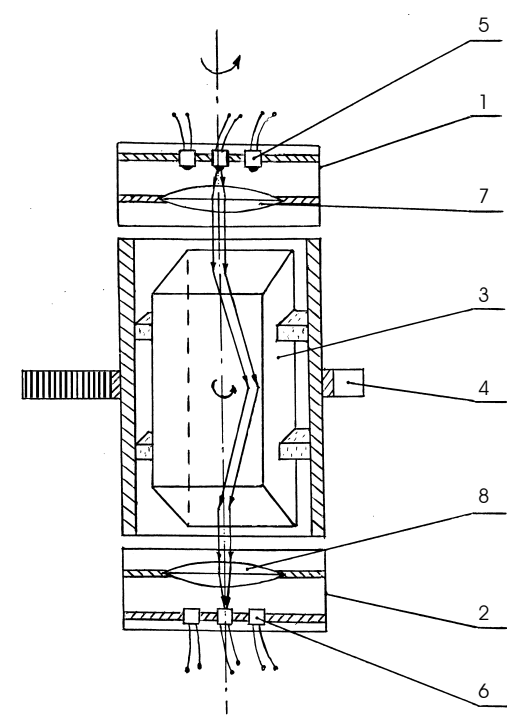

Fig. 1. Schematic view of the active optical rotary connector with the Dove prism and photoelectric transducers. 1 - rotary part; 2 - stationary part; 3 - Dove prism; 4 - reduction mechanism with the ratio $1: 2 ; 5$ - optical radiators (LEDs); 6 optical detectors (photodiodes); 7 - collimating lens; 8 focusing lens. 
The depicted in this figure multichannel optical rotary connector includes two parts - rotary $l$ and stationary $2-$ mounted on the common rotation axis as well as the compensator 3 placed in alignment between them and based on the Dove prism for turning the light beams. The Dove prism is mechanically related with the rotary part 1 via the tooth reducing motion 4 possessing the reduction ratio $1: 2$.

In every optical channel of ORC, placed on the rotary part 1 are the sources of radiation 5 (LEDs), while the detectors of radiation ( $p$ - $i$ - $n$-photodiodes) are mounted in the stationary part 2 . Besides, the rotary part 1 includes the collimating lens 7 , in the focal plane of which the LEDs 5 are located, while the stationary part 1 includes the focusing lens 8 with photodiodes 6 in its focal plane.

The lens 7 is intended to form parallel light beams at the input into the Dove prism, which is aimed at elimination of the influence of prism astigmatism on the formed image. The lens 8 focuses the optical beams at the prism output onto the respective photodetectors.

The Dove prism is an isosceles prism of right vision with one reflective and two refractive faces placed at the angles $45^{\circ}$ relatively to the reflective one [53, 54]. The prism has the mirror plane of symmetry, axis of symmetry of the first order and inversion center. When the prism is fixed, and entering beams turn around the optical axis, the prism inverts direction of beam turn. And while the prism turns around the optical axis, the input beams are turned at the output by the double angle as compared to that of the prism turn.

Owing to these properties, the prism can be used as an optical compensator of light beam rotation, if the prism turns with the speed two times less than that of beams. With this purpose, in the facility (Fig. 1) the reducing motion 4 with the reducing ratio $1: 2$ is used, which provides connection between the prism and rotary part 1 .

To determine the most important characteristics of this ORC (value of optical losses in the transmitting channels, depth of modulation in the optical signal amplitude when the prism turns around the axis, value of cross-interference between channels, and the maximum amount of optical channels available in this facility), we performed theoretical investigations of this facility behavior in various constructive solutions.

In what follows, we have considered the active ORC construction with optoelectronic transducers as well as the passive ORC construction with optical interfaces.

\subsubsection{Active construction of ORC with the Dove prism and collective lenses for focusing and collimation of beams}

Active construction of ORC with optoelectronic transducers at its inputs and outputs connected with electric transmission lines was created using thin nonaberrational elliptical lenses instead of the spherical ones. The former provide considerably lower aberrations as compared with biconvex thick lenses with spherical surfaces that were used in the facility [35].

The results of these investigations showed that application of a prism as a compensator of light beam rotation in ORC with collimating and focusing lenses, which are common for all the optical transmission channels, is related with a number of technical difficulties.

The matter is that direct-vision prisms, e.g. the Dove or Pehan ones, operate without astigmatism exclusively in parallel optical beams $[53,54]$, while the angular dispersion of beams from semiconductor light sources (laser diodes) used in wide-band channels for optical transmission comprises the range from several degrees up to several tens degrees. If using these light sources, the collimation lens cannot form a light beam with necessary parallelism, and the focusing lens is unable to provide necessary focusing on the detector. It is the reason for a considerable part of non-focused light to fall into adjacent receiving channels, which causes a noticeable cross-interference between channels.

To avoid this cross-interference caused by overlapping images created by adjacent light sources at the receiving side of ORC, the optical sources and respective optical detectors should be mounted at sufficient distances between them. At least, this distance should correspond to the dimension of the source image created by the lens in its focal plane. Due to increasing the distance between light sources, the component of cross-interference caused by overlapping between diffuse images can be considerably reduced. But moreover, in this case the total amount of optical detectors that can be mounted in the facility (i.e., optical channels, the amount of which corresponds to that of light sources).

At the first glance, it seems sufficient to increase transverse dimensions of the prism, which will result in the increased amount of channels. But since the dimension of the astigmatic image (scattering circle) grows with the prism dimensions, the amount of channels increased in this way grows only insignificantly. An optimal solution of this task needs a mathematical analysis that has been performed in the work [55].

Shown in Fig. 2 is the path of light rays in the facility for a point source in the principal plane of facility (in the mirror plane of prism symmetry).

The prism optical axis and principal optical axes of the lenses are related with the rotation axis. Light sources are placed along the circle in the focal plane of the collimation lens at the distance $h$ from its optical axis. Detectors are placed in the focal plane of focusing lens symmetrically to the light sources.

When deducing the equations describing the path of rays in the optical system shown in Fig. 2, we assumed that the light sources are point. To plot the images created by real sources, calculations are usually performed for a set of point sources placed along the peripheral contour line of source. 


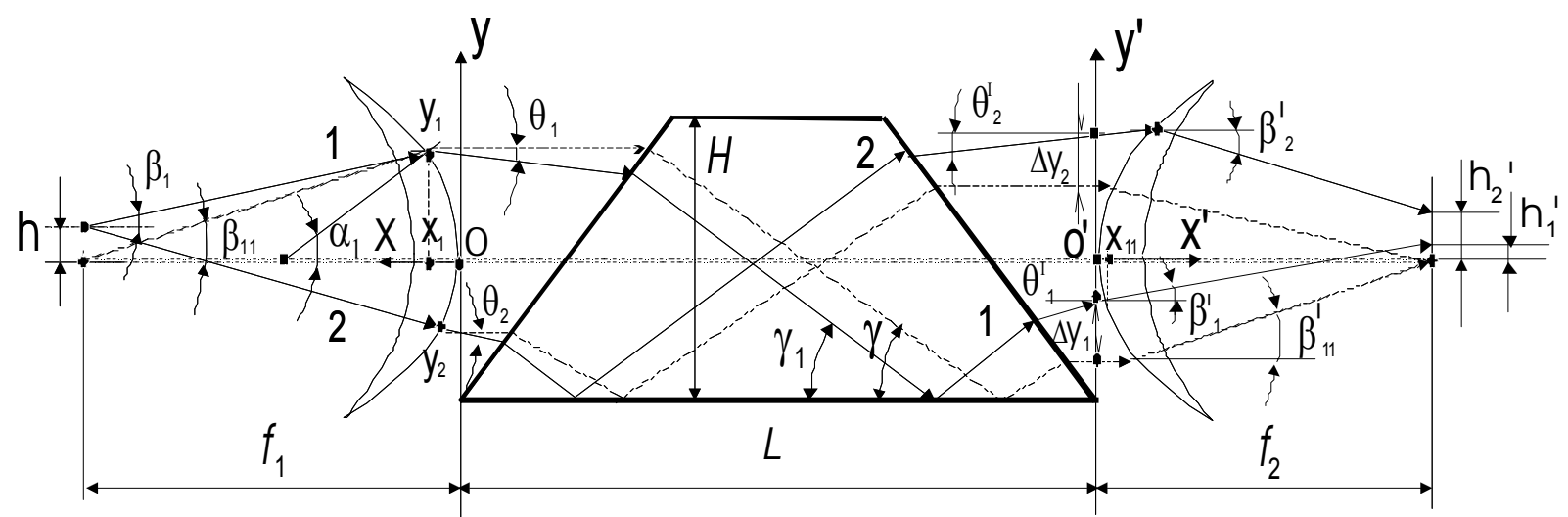

Fig. 2. The scheme of ray paths in ORC with the Dove prism and optical means (elliptical non-aberrational lenses) for focusing the rays.

\subsubsection{Mathematical model of the active ORC facility with the Dove prism and collective optical means for focusing the beams}

Solving the task of determination of ray paths can be separated by three stages. It means that light ray paths in collimation lens, Dove prism and focusing lens can be considered separately.

Let us consider the light ray paths in the meridional plane of the collimation lens.

Let us assume that the optical axis of the lens coincides with the $\mathrm{OX}$ axis, while the $\mathrm{OY}$ axis is directed along the perpendicular to the lens optical axis. The prism is placed in such a manner that its optical axis also coincides with the $\mathrm{OX}$ axis, and the $\mathrm{OY}$ axis of the coordinate system lies in the prism principal plane and passes through the middle of the prism edge along the perpendicular to it (Fig. 2).

The equation of projectively-equivalent surface for the elliptic lens in the rectangular coordinate system with its origin superposed on the lens apex, when the lens optical axis coincides with the OX axis, is as follows [56]:

$\left(1-1 / n_{1}^{2}\right) \cdot x^{2}-2 \cdot\left(1-1 / n_{1}\right) \cdot f \cdot x+y^{2}=0$,

where $f$ is the lens focal distance, $n_{1}$ - lens refraction index, and $x, y$ are the coordinates of the elliptic lens surface.

Let us write the equation for the ray going out of the point located in the lens focal plane at the distance $h$ from the OX axis

$y=h+(f-x) \cdot \operatorname{tg} \beta_{1}$,

where $\beta_{1}$ is the angle of incidence for the rays relatively to the $\mathrm{OX}$ axis.

Solving the expression (1) in combination with (2), one can obtain a quadratic equation, solution of which is the $x_{1}$ coordinate of intersection of the ray with lens

$x_{1}=\left(-b_{1}-\sqrt{b_{1}^{2}-4 a_{1} \cdot c_{1}}\right) / 2 a_{1}$, where

$$
\begin{aligned}
& a_{1}=1-1 / n_{1}^{2}+\operatorname{tg}^{2} \beta_{1}, \\
& b_{1}=2 f_{1} \cdot\left(1-1 / n_{1}+\operatorname{tg}^{2} \beta_{1}\right)+2 h \cdot \operatorname{tg} \beta_{1}, \\
& c_{1}=h^{2}+f_{1}^{2} \cdot \operatorname{tg}^{2} \alpha+2 h f_{1} \cdot \operatorname{tg} \beta_{1} .
\end{aligned}
$$

The $y_{1}$ coordinate for the same point can be found by substitution of the obtained $x_{1}$ value into the equation (2)

$y_{1}=h+\left(f-x_{1}\right) \cdot \operatorname{tg} \beta_{1}$.

Let us find the angle $\theta_{1}$ inherent to the ray refracted by lens relatively to the optical axis of the lens. To do it, one can use the non-aberrational property of the elliptic lens, physical essence of which is that the light rays, going out of the principal focal point of lens, propagate after refraction on its surface in parallel to its principal optical axis.

This condition allows finding the angle $\alpha$ between the straight line normal to the lens surface at the point $\left(x_{1}, y_{1}\right)$ and the OX axis, as well as to determine the angle $\theta$ for the rays going out of the lens, for the light source located off-axis by using the law of light refraction at the optical surface.

With account of the mentioned above, it can be shown that the angle $\theta_{1}$ for the refracted ray 1 can be found from the following relations:

$\theta_{1}=\arcsin \left[n_{1} \cdot \sin \left(\alpha_{1}-\beta_{1}\right)\right]-\alpha_{1}$,

$\alpha_{1}=\operatorname{arctg}\left(\frac{n_{1} \cdot \sin \beta_{11}}{n_{1} \cdot \cos \beta_{11}-1}\right)$,

$\beta_{11}=\operatorname{arctg}\left(\frac{y_{1}}{f_{1}-x_{1}}\right)$,

where $\beta_{11}$ is the slope angle for the ray relatively to the OX axis, when this ray goes out of the lens focal point to the point with coordinates $\left(x_{1}, y_{1}\right), \alpha_{1}-$ slope angle relatively to the $\mathrm{OX}$ axis for the normal to the lens elliptical surface at the same point $\left(x_{1}, y_{1}\right)$. 
By analogy, one can find the angle $\theta_{2}$ for another extreme ray (ray 2 in Fig. 2 with the maximum aperture), then he can determine the coordinates $y_{11}, y_{22}$ of the intersection points of respective rays with the OY axis

$y_{11}=y_{1}+x_{1} \cdot \operatorname{tg} \theta_{1}$,

$y_{22}=y_{2}+x_{2} \cdot \operatorname{tg} \theta_{2}$. prism.

Let us consider the light ray paths in the Dove

It should be noted that the rays falling onto the prism in parallel to it optical axis at the distance $h_{1}$ from it go out of the prism at the distance minus $h_{1}$ not changing their initial direction. The rays falling at the angle $\theta$ to the prism optical axis change their initial direction by that inherent to mirror reflection when going out of the prism. It is accompanied by ray shifts along the OY axis by the value $\Delta y$. This value consists of three components, namely: $\delta_{\mathrm{Y} 1}-$ for the air space at the input to the prism, $\Delta_{p r}-$ for the prism bulk, and $\delta_{\mathrm{Y} 2}-$ for the air space at the prism output.

The $\Delta y$ value for the ray total shift can be determined using the following expressions describing the ray paths in the prism:

$$
\begin{aligned}
& \Delta y=H \cdot \operatorname{tg} \theta+\Delta_{p r}+\Delta_{p r} \cdot \operatorname{tg} \theta, \\
& \Delta_{i ð}=H \cdot \frac{\operatorname{tg} \alpha \cdot\left(\operatorname{tg} \gamma-\operatorname{tg} \gamma_{1}\right)}{\operatorname{tg} \gamma \cdot\left(1+\operatorname{tg} \gamma_{1}\right)}, \\
& \gamma=45^{\circ}-\arcsin \left(\frac{\sqrt{2}}{2 \cdot n_{p r}}\right), \\
& \gamma_{1}=45^{\circ}-\arcsin \left(\frac{\sin \left(45^{\circ}+\theta\right.}{n_{p r}}\right),
\end{aligned}
$$

where $H$ is the height of the Dove prism, $n_{p r}$ - prism refraction index, $\theta$ - slope angle for the ray falling onto the prism relatively to the OX axis, $\gamma, \gamma_{1}$ are the angles of incidence for the rays relatively to the prism base (Fig. 2), $\alpha$ is the angle between the prism input face and its base, which is equal to $45^{\circ}$ in the Dove prism.

In Fig. 2, for the upper ray $\theta=\theta_{1}$, while for the lower one $\theta=\theta_{2}$. The sign for the angle $\theta$ is chosen using the general rule. In our case, this sign is negative for both rays.

Let us consider the light ray paths in the meridional plane of the focusing lens.

Let a new rectangular coordinate system $\mathrm{X}^{\prime} \mathrm{O}^{\prime} \mathrm{Y}^{\prime}$ has its $\mathrm{O}^{\prime} \mathrm{X}^{\prime}$ axis directed along the principal optical axis of another elliptic lens and its $\mathrm{O}^{\prime} \mathrm{Y}^{\prime}$ axis directed along the normal to the lens and prism optical axes and passing through the middle of the edge between the prism output refractive face and its base (reflective face). The apex of this elliptic lens is located in the origin of the coordinate system $\mathrm{X}^{\prime} \mathrm{O}^{\prime} \mathrm{Y}^{\prime}$.

Having determined the $\Delta y_{1}$ and $\Delta y_{2}$ values for the ray shifts at the prism output, one can find the coordinates $y_{11}^{\prime}$ and $y_{22}^{\prime}$ of the intersection points for the rays with the $\mathrm{O}^{\prime} \mathrm{Y}^{\prime}$ axis that lies in the plane $x^{\prime}=0$

$$
\begin{aligned}
& y_{11}^{\prime}=y_{11}+\Delta y_{1}+x_{11} \cdot \operatorname{tg} \theta_{1}, \\
& y_{22}^{\prime}=y_{22}+\Delta y_{2}+x_{22} \cdot \operatorname{tg} \theta_{2} .
\end{aligned}
$$

Further, one can determine the coordinates $x_{1}^{\prime}, y_{1}^{\prime}$ and $x_{2}^{\prime}, y_{2}^{\prime}$ inherent to the points of intersection between these rays and lens surface. Here, it is pertinent to use Eq. (1) for the surface of elliptic lens in the new coordinate system $\mathrm{X}^{\prime} \mathrm{O}^{\prime} \mathrm{Y}^{\prime}$ as well as equations for rays going out of the points $y^{\prime}{ }_{11}$ and $y_{22}^{\prime}$ identical to Eq. (2).

For the ray going out of the point $y_{11}^{\prime}$, one can obtain the following relations:

$$
\begin{aligned}
& x_{1}^{\prime}=\frac{-b_{2}-\sqrt{b_{2}^{2}-4 y_{11}^{\prime 2}\left(1-1 / n_{2}^{2}+\operatorname{tg}^{2} \theta_{1}\right)}}{2\left(1-1 / n_{2}^{2}+\operatorname{tg}^{2} \theta_{1}\right)}, \\
& y_{1}^{\prime}=y_{11}^{\prime}+x_{1}^{\prime} \cdot \operatorname{tg} \theta_{1},
\end{aligned}
$$

where $\quad b_{2}=2 f_{2}\left(1-1 / n_{2}+\operatorname{tg}^{2} \theta_{1}\right)+2 y_{11} \cdot \operatorname{tg} \theta_{1}, \quad n_{2}-$ refraction index for the focusing lens, $f_{2}$ - focal distance of this lens.

For the second ray, there exist analogous relations where, instead of the angle $\theta_{1}$ and ordinate $y_{11}^{\prime}$, the angle $\theta_{2}$ and ordinate $y_{22}^{\prime}$ take place.

The light rays 1 and 2 after refraction at the external surface of the focusing lens further propagate under the angles $\beta^{\prime}{ }_{1}$ and $\beta^{\prime}{ }_{2}$ relatively to the lens optical axis and intersect the lens focal plane in the points located at the distances $h_{1}^{\prime}$ and $h_{2}^{\prime}$ from its optical axis, respectively.

The values of angles $\beta^{\prime}{ }_{1}$ and $\beta^{\prime}{ }_{2}$ as well as distances $h^{\prime}{ }_{1}$ and $h^{\prime}$ can be calculated using the following equations:

$$
\begin{aligned}
& \beta_{1}^{\prime}=\alpha_{1}^{\prime}-\arcsin \left[\sin \left(\alpha_{1}^{\prime}+\theta_{1}\right) / n_{2}\right], \\
& \alpha_{1}^{\prime}=\operatorname{arctg}\left(\frac{n_{2} \cdot \sin \beta_{11}^{\prime}}{n_{2} \cdot \cos \beta_{11}^{\prime}-1}\right), \\
& \beta_{11}^{\prime}=\operatorname{arctg}\left(\frac{y_{1}^{\prime}}{f_{2}-x_{1}^{\prime}}\right), \\
& \left\{\begin{array}{c}
h_{1}^{\prime}=y_{1}^{\prime}-\left(f_{2}-x_{1}^{\prime}\right) \cdot \operatorname{tg} \beta_{1}^{\prime} \\
h_{2}^{\prime}=y_{2}^{\prime}-\left(f_{2}-x_{2}^{\prime}\right) \cdot \operatorname{tg} \beta_{2}^{\prime}
\end{array}\right\} .
\end{aligned}
$$

The difference $\left(h^{\prime}{ }_{1}-h_{2}^{\prime}\right)$ is the highest dimension of the image spot in the principal optical section of a facility (in the direction of $O Y^{\prime}$ axis) in the lens focal plane, this section being created by the rays with the highest aperture.

When the prism rotates, the image spot also moves - precesses around some point $h^{\prime}$. Therefore, when the facility operates, the maximum radius of spot scattering is defined by the highest distance between the point, around which this precession is realized, and the points $h_{1}^{\prime}$ or $h_{2}^{\prime}$. 
2.1.3. Results of theoretical studying the active ORC with the Dove prism and lens collimating optics common for all optical channels

Using the obtained above expressions (1) to (21), in [55] we performed calculation for the radius of the confusion circle as a function of the aperture inherent to point source radiation for the number of real construction parameters of facility.

Fig. 3 shows a family of calculated dependences for the diameter of confusion circle on the distance $h$ between the point radiation source and the lens optical axis for elliptic lenses with the focal distances $f=25,50$, 75 and $100 \mathrm{~mm}$. The refraction index of the prism and lenses was assumed to be equal to 1.7. The aperture angle value of the radiation source was $\beta=6^{\circ}$.

When performing calculations, the height of the prism $H$ was chosen as the minimum possible one for the set aperture angle $\beta$ of the optical source and focal distance of lenses by using the condition that highaperture rays should not exceed the transverse dimensions of the prism and propagate outside it.

Also, there is a limitation for the acceptable prism dimension. The rays should propagate inside the prism without any vignetting. However, when these dimensions are large this condition is not performed. The rays incident onto the prism at various angles, during their propagation inside the prism, obtain at the prism output some additional shift $\Delta y$ (see Exps (10) to (13)) proportional to the prism height and the angle $\theta$ of ray incidence onto the prism.

In its turn, the angle $\theta$ is in proportion to the distance between the source and axis (see Exps (5) to (7)). When the prism height is larger than some critical value or the source is located off-axis above some critical distance, then inclined rays inside the prism obtain so considerable additional shift $\Delta_{p r}$ that after reflection from the prism base they fall not onto the output face but onto the upper face of the prism oriented in parallel to the base, and go out of the prism.

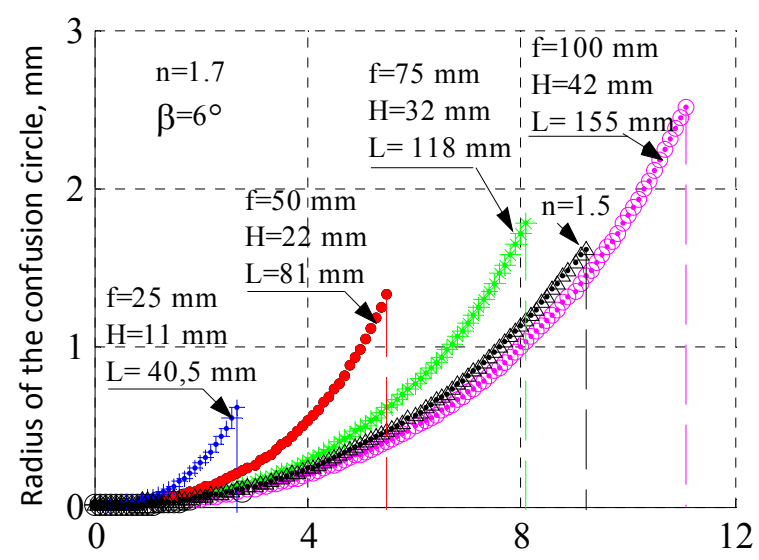

Distance $h$ of the point source to the optical axis, $\mathrm{mm}$

Fig. 3. Calculated dependences for the radius of radiation confusion circle on the distance $h$ of the point source to the optical axis of elliptical lenses with various focal distances $f$.
It is noteworthy that with increasing the distance between the light source and prism axis there arises a situation when the transfer of rays through the prism become impossible without vignetting for any arbitrary prism dimensions. Therefore, when calculating the maximum possible amount of channels in ORC the prism height was chosen with account of the condition that the point source is located at the maximum possible distance from the axis, for which the rays pass inside the prism yet.

Calculations of the limiting distance between the point source and axis as well as the choice of the prism height for this distance were performed using the method of step-by-step approximation. The distance source-toaxis was increased successively with the step $0.1 \mathrm{~mm}$ up to the moment when further increasing by this step resulted in impossibility for rays to pass through the prism without vignetting.

The calculated acceptable limiting distances $h$ between the source and axis for various lens focal distances $f$ and optimal height of the Dove prism are shown in Fig. 3 with dash-dotted lines drawn to the horizontal axis of the figure. Fig. 4 shows the dependences for the radius value of the confusion circle on the angle of ray incidence for various distances between the point source and axis in the facility with lenses of the focal distance $f=50 \mathrm{~mm}$.

To obtain real practical results, let us consider these dependences by using the available elemental base with account of existing discrete semiconductor radiation sources.

Shown in Figs 5a-5d are the contours of light beams for the light source of the type L3989-01 (Hamatsu) and contour lines of images at the facility output, which are created by elliptic lenses with various focal distances. The certified value of the aperture angle $\beta$ inherent to the LED L3989-01 is $6^{\circ}$. When calculating the maximum possible distances $h$ between this point source and axis (see Fig. 4), the angle of ray incidence was assumed to be $6^{\circ}$, too.

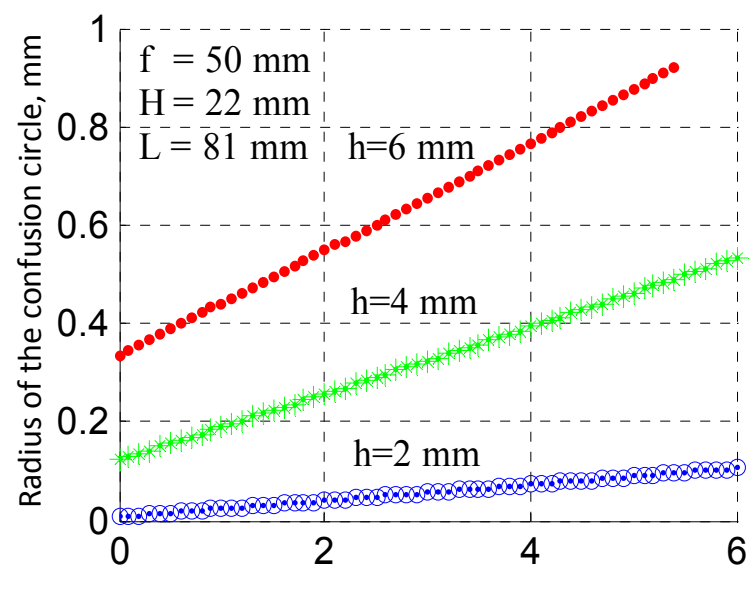

Angle of incidence for the source rays, deg.

Fig. 4. Calculated dependences for the radius of radiation confusion circle on the aperture angle $\beta$ of rays for various distances $h$ of the point source to the optical axis of elliptical lens. 
Table 1. The maximum possible amount of optical channels in ORC with LEDs of different types.

\begin{tabular}{|c|c|c|c|c|c|c|c|c|c|c|c|c|}
\hline \multirow{3}{*}{$\begin{array}{l}\text { (LED type) } \\
\text { Aperture angle } \beta\end{array}$} & \multicolumn{12}{|c|}{ Focal distance of the lens $f, \mathrm{~mm}$} \\
\hline & \multicolumn{3}{|c|}{25} & \multicolumn{3}{|c|}{50} & \multicolumn{3}{|c|}{75} & \multicolumn{3}{|c|}{100} \\
\hline & $N$ & $\begin{array}{c}\mathrm{H}, \\
\mathrm{mm}\end{array}$ & $\begin{array}{c}L, \\
\mathrm{~mm}\end{array}$ & $N$ & $\begin{array}{c}\mathrm{H}, \\
\mathrm{mm}\end{array}$ & $\begin{array}{c}L, \\
\mathrm{~mm}\end{array}$ & $N$ & $\begin{array}{c}\mathrm{H}, \\
\mathrm{mm}\end{array}$ & $\begin{array}{c}L, \\
\mathrm{~mm}\end{array}$ & $N$ & $\begin{array}{c}H, \\
\mathrm{~mm}\end{array}$ & $\begin{array}{c}, \\
\mathrm{mm}\end{array}$ \\
\hline$(\operatorname{SV} 5637-001) \beta=2^{\circ}$ & 1 & 8 & 30 & 7 & 18,5 & 69 & 14 & 29.6 & 109 & 21 & 37.5 & 140 \\
\hline$(\mathrm{SV} 2637-001) \beta=4^{\circ}$ & 1 & 11 & 40 & 5 & 20 & 74 & 10 & 31 & 114 & 17 & 41 & 151 \\
\hline$(\mathrm{L} 3989-01) \beta=6^{\circ}$ & 1 & 11 & 40 & 2 & 22 & 81 & 6 & 32 & 118 & 9 & 42 & 155 \\
\hline
\end{tabular}

In what follows, the obtained data for the maximum acceptable $h$ values (Fig. 4) were used to calculate coordinates for location of centers of real sources L3989-01, which were determined as the difference $(h-r)$, where $r$ is the radius of the case of this light source.

In Figs 5a-5d, the annular lines shown in diagrams with dots are related to dimensions of the LED case, the diameter of which is close to $5.4 \mathrm{~mm}$. The lines designated with small rings are related to the diameter of source light beam equal to $3.72 \mathrm{~mm}$ in accord with the LED L3989-01 certificate. The peripheral lines looking as ellipses plotted with asterisks (Figs. 5a-5d) are related to calculated contour lines for source images on the receiving side of facility.

The contour lines of optical images for light sources on the receiving part of ORC are plotted using superposition of images corresponding to point light sources located along the contour of radiator at different distances from the rotational axis.

Using the method described above for each variant of chosen lenses with different focal distances, we calculated optimal dimensions for the prism in such a manner that they provide to reach the largest amount of channels in this facility, when cross-interference between these channels is practically absent. As seen from Fig. 5, in the case of LED L3989-01 the maximum amount of optical channels for the lenses with focal distances 50,75 and $100 \mathrm{~mm}$ is equal to 2, 6 and 9 channels, respectively. Concerning the lens with the focal distance $25 \mathrm{~mm}$, the respective facility can contain only one channel located on the axis of rotation (diagram for this case is not shown in Fig. 5).

The results of calculations aimed at determination of the maximum possible amount of channels $N$ for the discussed above light sources in dependence on lens dimensions are adduced in Table 1 . The represented data show that the maximum amount of optical channels that can be reached in ORC with the Dove prism, when all the channels use the same ways for collimation and focusing the beams, depends on the light source aperture angle and dimensions of the ORC optical system. In particular, in the case of using the elliptical lenses with the focal distance $100 \mathrm{~mm}$ and the source with the aperture angle $\beta=2^{\circ}$, for example LED SV5637-001, the amount of channels can reach 21, while for the source with $\beta=6^{\circ}$ - only 9 channels.

However, it should be noted that in the case of using lenses with the focal distance $100 \mathrm{~mm}$, the total length of optical section in ORC (prisms with lenses) exceeds $355 \mathrm{~mm}$. It is clear that these dimensions are not suitable for many practical applications of these facilities.

For the lenses with the focal distance $50 \mathrm{~mm}$, the length of optical section can be reduced down to $180 \mathrm{~mm}$, but the maximum amount of channels in this case will be only 7 channels for the sources with the aperture angle $\beta=2^{\circ}$ and only 2 channels when this angle will be $\beta=6^{\circ}$. If the lens focal distance is lower than $25 \mathrm{~mm}$, then in this facility only one optical channel on the axis of rotation can be mounted.

The value of optical losses can be estimated if using high-frequency photodetectors possessing the frequency band $100 \mathrm{MHz}$ as an example. As a rule, these photodetectors have the diameter of photosensitive area no larger than $1 \mathrm{~mm}$. It is clear from Fig. 5 that the diameter of light spot from the light source at the plane of photodetector reaches approximately $8 \mathrm{~mm}$. Thereof, rude estimation of the optical loss value is close to $18 \mathrm{~dB}$, which is caused by mismatch between the diameters of photodetector and light beam incident on this detector.

The obtained results of calculations, performed for the active ORC construction based on the compensation principle with the Dove prism and general for all the transmission channels way of collimation and focusing the beams, enables to draw the following conclusions:

1. The maximum amount of channels in this facility reaches practically the value approximately 10 channels, because of inadmissibly large sizes and mass of a facility with larger amount of channels. In particular, the length of the optical system in the 10-channel ORC reaches approximately $300 \mathrm{~mm}$ and grows in proportion to the amount of channels. 
2. In the facility with the optical system of the length shorter than $70 \mathrm{~mm}$, only one optical channel mounted on the axis of rotation can be available.

3. In the facility even with ideal connection between channels, there is noticable cross-interference caused by light scattering on non-homogeneities inside the prism and optical surfaces of prism and lenses, the value of which is in proportion to the amount of channels.

These conclusions also concern other constructions of the ORC with prismatic compensators, in particular, the known from [35] ORC based on the Pehan prism, since astigmatism and aberrational image blurring in this prism are not lower than those in the Dove one.

\subsection{Passive construction of ORC with the Dove prism and collective lenses for focusing and collimation of beams}

The course of rays in the construction of ORC with fiber-optic transmission lines was calculated using the mathematical solutions obtained by the methods of analytic geometry, being based on geometric optics laws for reflection and refraction of light at optical surfaces. The value of optical losses in the transmission channels was computed using the method of numeric integration based on Fresnel equations. The respective program for modeling the facility was developed using the software package Mathlab.

Adduced below are the results of studying ORC made in the variant when its transmission and receiving parts are equipped with optical light guides serving as radiators and receivers.

Shown in Fig. 6 are the calculated dependences of the optical loss value in ORC transmission channels on the butt radius of the receiving light guide for various distances $h$ of this butt from the rotation axis. The calculations were performed for two cases, namely: i) in the absence of prism angular beating; ii) when the prism angular beating reaches the value $\Delta=0.06^{\circ}$.

Fig. 7 shows the calculated dependences of the optical loss value in the ORC optical channel on the prism angular beating for various values of the receiving light guide butt: 50, 100, 200 та $400 \mu \mathrm{m}$. These

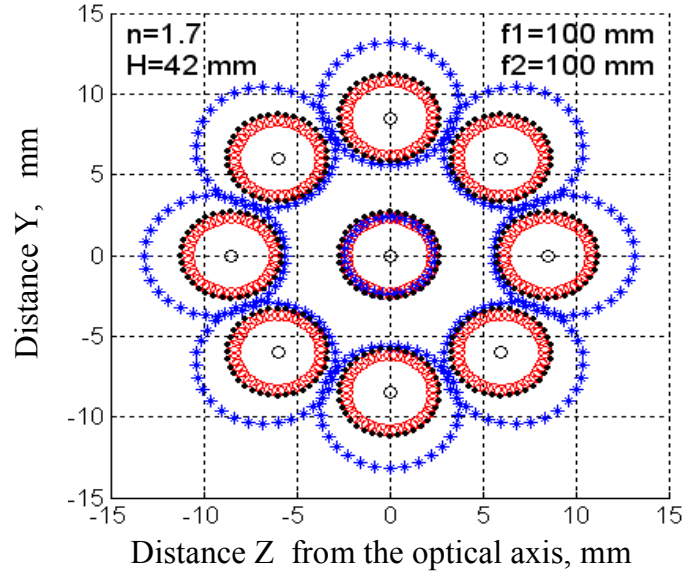

(a)

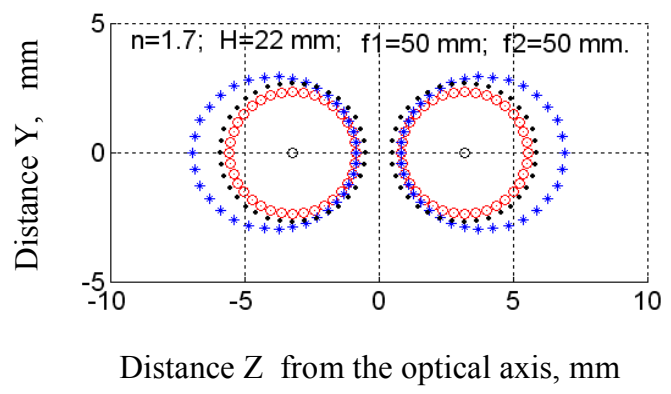

(c)

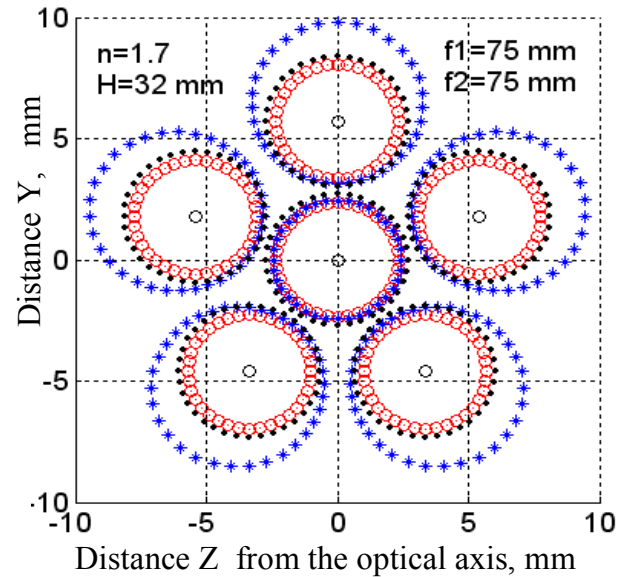

(b)

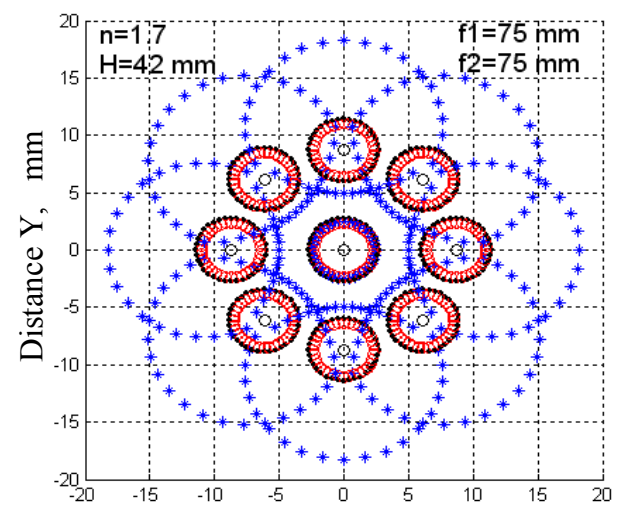

(d) Distance $\mathrm{Z}$ from the optical axis, $\mathrm{mm}$

Fig. 5. Diagrams for location of radiation sources L3989-01 on the ORC transmitting part and their contoured images within the plane of detectors on the receiving part, which are created by the ORC optical system based on the Dove prism and elliptical lenses with different focal distances. 
calculations were performed for spherical lenses with the following parameters: lens thickness is equal to $10 \mathrm{~mm}$, focal distance $-f=50 \mathrm{~mm}$, refraction index of the prism and lenses $-n=1.7$. The butt diameter and numeric aperture of the transmitting light guide were assumed to be typical for multichannel fiber-optic cables: the butt diameter $-50 \mu \mathrm{m}$, numeric aperture $-\mathrm{NA}=0.2$.

To minimize the sizes of the aberration scattering circle, the height of prism was chosen as low as possible for the given aperture of input beams and sizes of the collimating lens. The height was calculated with account of the condition that the extreme rays at the output of the collimating lens should not be blinded by the prism. For the above set lens and light guides characteristics, the optimal prism sizes were as follows: the height $-27 \mathrm{~mm}$, and length $100 \mathrm{~mm}$.

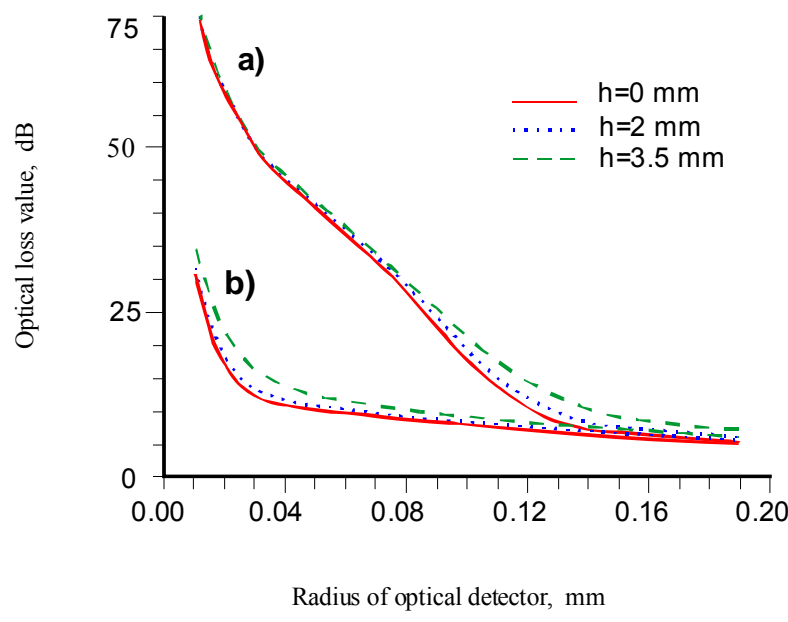

Fig. 6. Calculated dependences of optical loss values in the ORC optical channels with the Dove prism on the radius of receiving channel. a) $\Delta=0^{\circ}$; b) $\Delta=0.06^{\circ}$.

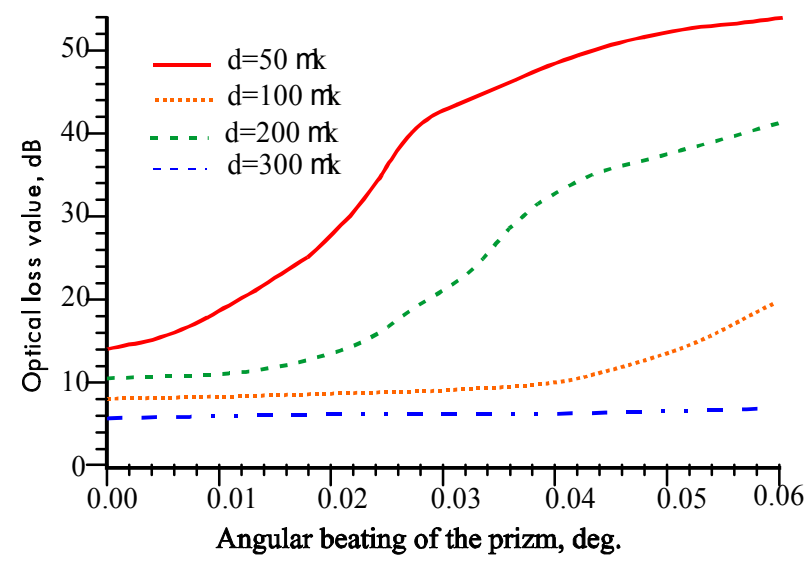

Fig. 7. Calculated dependences of optical loss values in the transmitting optical channel located on the rotation axis $(h=0)$ on the value of the prism angular beating for various values of the butt diameter $d$ in the receiving light guide.
As seen from Figs 6 and 7, the essential deficiencies of this ORC are high optical losses and considerable oscillations of the signal amplitude during prism rotation, which is caused by prism beating. For instance, when prism angular beating is as low as $0.06^{\circ}$, modulation rotational oscillations of the optical signal amplitude reach the value $30 \mathrm{~dB}$ for the light guide of $100-\mu \mathrm{m}$ diameter and $40 \mathrm{~dB}$ for that of $50-\mu \mathrm{m}$ diameter. Optical losses for the above light guides are 40 and $54 \mathrm{~dB}$, respectively. If one takes into account the losses by Fresnel for this case as well as the losses caused by non-ideal mutual arrangement of lenses and light guides in ORC transmitting channels, the total losses will exceed $50 \mathrm{~dB}$ in the first case and $60 \mathrm{~dB}$ in the second one. Thus, such facility is unfit to be used in circuits of fiber-optic transmission lines with standard cables.

Thereof, the ORC construction with collective lenses for focusing and collimation of beams can be used in the multichannel variant only as an active construction in the composition with electrical transmission lines. In the passive variant with optical interfaces based on light guides without additional focusing and collimating lenses, there take place inaccessibly high optical losses. In particular, for the standard optical cables with the core diameter $50 \mu \mathrm{m}$, the value of optical losses exceeds $36 \mathrm{~dB}$, and for the non-standard cables with the core diameter $100 \mu \mathrm{m}$ this value exceeds $30 \mathrm{~dB}$.

\section{Multichannel ORC with a mirror compensator}

When creating the multichannel ORC with a mirror compensator, the mirror needs to satisfy two specific requirements: it should be double-sided and as far thin as possible. To make a facility with such mirror and, being more exact, to mount it in such a manner that external harmful factors (vibrations, temperature) could not have any effect on it, is very difficult. In the most optimal and original form, these tasks are solved in the technical solution patented by [44]. In their facility schematically depicted in Fig. 8, the optical compensator 4 consists of two optical hemispheres separated between each other with the thin double-sided mirror 5 that is not affected by any external harmful factors.

The optical compensator 4 is mounted on the rotation axis 3 between the rotor 17 and stator 16 through the insert 9 . The rotational axis of the ORC rotor 3 passes through the center of the optical sphere and lies within the plane of the mirror 5. The insert 9 is articulationly joined with the case 6 due to bearings 10 and coupled with inserts 16,17 via the conical gears 12 , 14,15 possessing the reduction coefficient 1:2.

Two groups of optical fibers (transmitting 1A-1N and receiving $2 \mathrm{~A}-2 \mathrm{~N}$ ) are located in the respective inserts 16, 17 along the arc around the compensator sphere and are oriented relatively to the mirror in such a way that the butts of one group are the mirror images for respective butts of another group. The lenses 18 serve as 
the collimator ones for the transmitting group and as the focusing ones for the receiving group.

The insert 16 is placed in the case 6 , while the insert 17 - in the case 7 that is articulationly joined with the case 6 via the bearings 8,11 , which enables it to freely rotate around the axis 3 , together with the insert 17 and a group of light guides $2 \mathrm{~A}-2 \mathrm{~N}$ mounted in it.

When the insert 17 together with the group of light guides $2 \mathrm{~A}-2 \mathrm{~N}$ rotates by an arbitrary angle $\varphi$ relatively to the insert 16 , the optical sphere 4 with the mirror 5 also rotate in the same direction by the halved angle $\varphi / 2$ due to a gear mechanism.

Compensation of the beam turn in the facility is reached by the following way.

Let us assume that, in one of the azimuthal positions of the insert 17 relatively to the insert 16 , the light guide group $2 \mathrm{~A}-2 \mathrm{~N}$ is optically connected with that $1 \mathrm{~A}-1 \mathrm{~N}$ due to the mirror 5 .

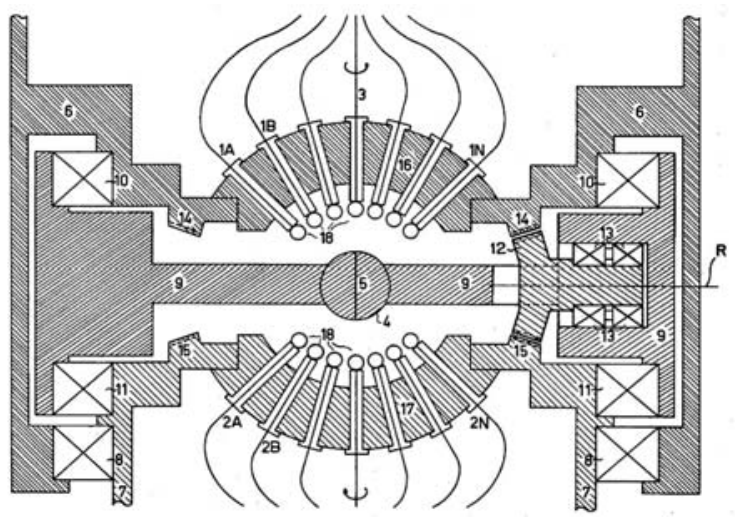

Fig. 8. Optical rotary connector with mirror compensator [44]: $1 \mathrm{~A}-1 \mathrm{~N}$ - group of transmitting light guides; $2 \mathrm{~A}-2 \mathrm{~N}$ - group of receiving light guides; 3 - axis of rotation; 4 - optical sphere; 5 - double-sided mirror.

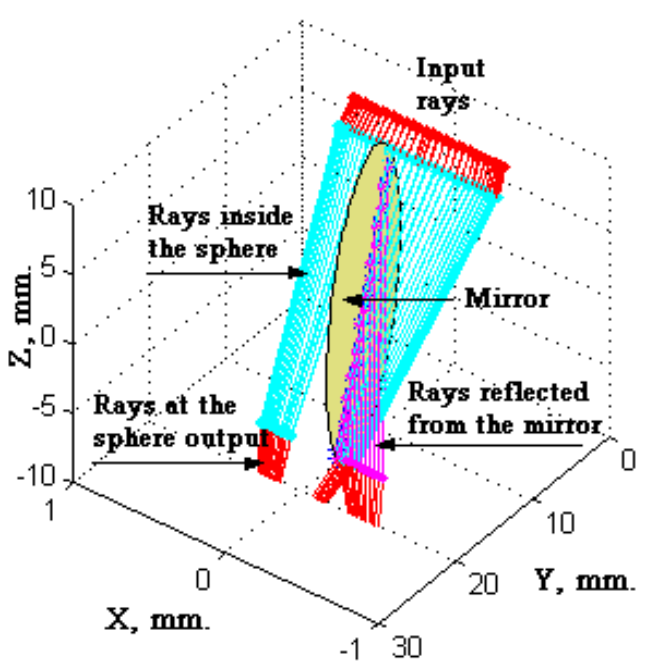

Fig. 9. Paths of rays in ORC for one of mirror positions, when the mirror crosses the input light flow.
When the mirror does not move, and light guides $2 \mathrm{~A}-2 \mathrm{~N}$ with the insert 17 turn by the arbitrary angle $\varphi$, the beams radiated by the light guides $2 \mathrm{~A}-2 \mathrm{~N}$ are reflected by the mirror in the direction opposite to the rotation direction of the incident beams by the angle " $-\varphi$ ". Since the mirror also rotates, then its turn by the angle $+\varphi / 2$ causes the turn of the reflected beams by the angle $+\varphi$. As a result, at the output of the optical sphere the position of beams remains unchanged. In other words, there takes place compensation of beam movement, and from a theoretical viewpoint the facility is functionally capable.

At the same time, when thoroughly considering operation of this facility there arises a controversial question related with the possibility to avoid here significant optical losses as well as considerable rotational vibrations in the value of optical losses in those mirror angular positions when the mirror crosses the input light flow and separate it by two ones (see Fig. 9). It causes the following question: is it allowed to practically use the facility with these deficiencies?

Since the description for the USA patent №4447114 does not contain any optical characteristics of the respective facility, to determine them in [57] there were performed detailed theoretical investigations of this facility behavior by using the method of computer modelling.

Studying the facility was performed for two in principle different variants of its realization. First, in the variant with fiber-optic collimators that provide a wide collimated optical beam with the diameter 1 to $4 \mathrm{~mm}$. These collimators are designed being based on fiber-optic light guides and quarter-wave gradient plates or spherical lenses. Second, in the variant with fiber-optic collimator that provides a narrow optical beam with the diameter lower than $0.2 \mathrm{~mm}$. The latter can be created on the base of single-mode light guides and spherical microlenses.

Fig. 9 illustrates the calculated beam course in ORC. There used is the constructive variant with wide collimated light beams. To image in one figure the focused optical patterns of small sizes, which are created by light beams at the output of optical sphere, as well as the course of beams through the constructive elements of considerably larger sizes (optical sphere with the mirror, focusing and collimating lenses) this sphere and mirror are depicted in Fig. 9 in a slightly deformed look. Along the $\mathrm{X}$ axis, the picture dimensions are elongated, while along the $Z$ axis they are squeezed. Besides, for simplicity Fig. 9 shows only those input rays that contour the light flow incident on the optical sphere from the lens fiber-optic collimator.

Calculations of ray courses were performed in the facility model with the following parameters: the radius of optical sphere $R=10 \mathrm{~mm}$, refractive index of the sphere $n=1.5$, diameter of input light beam $d=1 \mathrm{~mm}$, aperture angle of the input light beams $\Psi=0.6^{\circ}$. The diameter of light beam and aperture of rays are taken as those for typical multi-mode light guides with the diameter $50 \mu \mathrm{m}$ and the collimating gradient quarterwave lens with the diameter $1 \mathrm{~mm}$. 


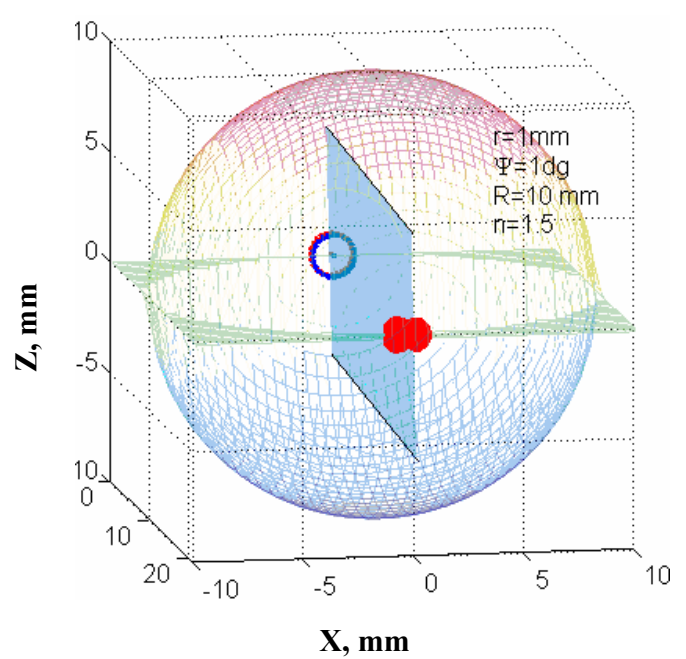

Fig. 10. 3D image of the optical sphere, mirror, input light beam of ring shape, and spot of focused beams at the output of optical sphere.

To provide more obvious reception, shown in Fig. 10 are the 3D image of the optical sphere, plain mirror, contour of the collimated optical beam possessing the ring shape at the sphere input and the light beam at the sphere output, which is split by the double-sided mirror. This figure is plotted when calculating the facility with the following parameters: radius of the optical sphere $R=1 \mathrm{~mm}$, refraction index of the sphere $n=1.5$; radius of the input beam $r=1 \mathrm{~mm}$; aperture angle of the collimated beams $\Psi=1^{\circ}$.

As seen from Figs 9 and 10 in angular positions of the mirror when it splits the input light beam the part of rays inside the optical sphere is reflected from the mirror, while another part freely passes along the mirror. At the output of optical sphere, the light beam is separated by several light beams. The beams reflected from the mirror in accord with the mentioned above principle of compensation are focused onto the butt end of receiving light guide, while the beams that pass along the mirror do not change their initial direction. It is obvious that in above mentioned positions of the mirror in the facilities with the mirror compensator there take place specific, inherent to these constructions optical losses of signals as well as rotational dependence of the transfer coefficient for the optical signal, when the mirror rotates.

Investigated in [57] was the influence on ORC optical characteristics from various constructive factors, namely: the diameter and refractive index of the optical sphere, diameters and refractive indexes of spherical collimating and focusing lenses, diameter of light guide core and its numeric aperture. Also, the authors studied the influence ORC characteristics from exactness of manufacturing the construction elements and their composition, which plays the principal role in determination of technological effectiveness inherent to the facility.

The method of calculations performed in [57] for the transfer coefficient and the value of optical losses can be briefly described as follows.
The optical losses between the butts of transmitting and receiving light guides were calculated with account of the ratio between the number of beams focused on the butt of receiving light guide and total amount of beams radiated by the transmitting light guide, taking into account the intensity of each beam and numeric aperture NA of light guides.

The intensity of beams was determined in assumption that the surface brightness of light at the butt of light guide is homogeneous over the butt area and obeys the Lambert law, which is close to the real distribution of the light intensity at the output butt of a light guide, when one uses a light source with a high aperture, for instance, LED.

The source of radiation (in our case, it is the radiating butt of a light guide) was separated by $l$ ring zones, each of them, in its turn, was separated by $m$ sectors. Centers of these sectors were defined as points (coordinates) of output for homocentric light beams, the intensity of which was conditionally considered as the total intensity that can be radiated by the respective sector of the light guide butt in all the possible directions with the boundary angle corresponding to the light guide numeric aperture.

In its turn, the homocentric beams from each sector were separated by $n$ conical beams each of them was additionally separated by $g$ azimuthal sectors.

Thus, after partitioning the light beam by the elementary ones the intensity of one beam was considered as the intensity that corresponds to radiation from the elementary area of the light guide butt in a set direction within the limits of an elementary solid angle. Before calculation of the intensity for this elementary beam $P_{\text {elem }}$, they calculated the total intensity $P_{i k}$ of all the beams that propagate between two conical surfaces from the following mathematical expression [58]:

$$
\begin{aligned}
& P_{i k}=2 \pi \cdot I \cdot\left[\cos \left(\alpha_{i}\right)-\cos \left(\alpha_{k}\right)\right], \\
& I=I_{0} \cdot \cos \left(\alpha_{i}\right),
\end{aligned}
$$

where $I_{0}$ is the intensity of light beam along the cone axis, $\alpha_{i}$ and $\alpha_{k}$ are the plain angles at the apexes of respective cones.

The intensity of one beam was calculated using division of the total intensity of beams $P_{i k}$ on the amount of ring zones and sectors corresponding to the number of elementary parts with account of their areas by using the following mathematical relation

$$
P_{\text {elem }}=P_{i k} \cdot\left(r_{q}^{2}-r_{q-1}^{2}\right) / r^{2} \cdot m \cdot g,
$$

where $r$ is the light guide butt radius, $r_{q}$ and $r_{q-1}$ are external and internal radii of the respective butt ring zone.

When the light guide butt surface is divided by sufficiently small areas of elementary sectors and the solid angle is divided by elementary solid angles, then the accuracy of calculations for the transfer coefficients can be made as high as one likes. Using the iteration method, one can ascertain that, to reach the accuracy of calculations at the level $0.2 \%$, it is sufficient to choose $l=10, m=36, g=36, \alpha_{i}=\alpha_{k}=0.25^{\circ}$. 


\subsection{Optical losses and rotational dependence for the coefficient of optical transfer inherent to ORC with mirror compensator and fiber-optic collimator, which provide wide optical beams}

Depicted in Fig. 11 are the angular dependences for the ORC transfer coefficients calculated for the facility with the optical sphere of the radius $R=10 \mathrm{~mm}$ with singlemode and multimode light guides in optical receiving channels. These calculations were performed for ORC with ideally exact geometry of construction (transverse shift of the light guide butt $\Delta x$, transverse shift of the sphere center $\delta X_{c}$ and angular beating $\Delta \beta$ of the mirror in the horizontal plane were assumed to have zero value).

At the transmitting side of the optical channel, fiber-optic collimators are used, they are based on a spherical lens of the radius $r=2 \mathrm{~mm}$.

As to the receiving side, we considered two variants of: multimode fiber-optic light guide $(d=50 \mu \mathrm{m}, \mathrm{NA}=0.19)$ and single-mode light guide $(d=7 \mu \mathrm{m}, \quad \mathrm{NA}=0.13)$. The focusing lenses were assumed to be analogous to the collimating ones $(r=2 \mathrm{~mm}, n=1.5)$.

It is seen from Fig. 11 that in angular positions of the mirror $\beta$ when it crosses the light beam (near $90^{\circ}$ in the figure) one can observe a sharp drop of the transfer coefficient down to the value lower than $5 \%$. In this moment, the value of modulation amplitude reaches $95 \%$.

The angle $\beta$ is conditionally assumed to be equal to $90^{\circ}$ for the mirror position, when its normal lies along the perpendicular to the plane where the axes of transmitting and receiving light guides lie, these guides being placed along the ring around the compensator optical sphere (Fig. 9). In other words, when the mirror plane and the plane where axes of light guides lie coincide.

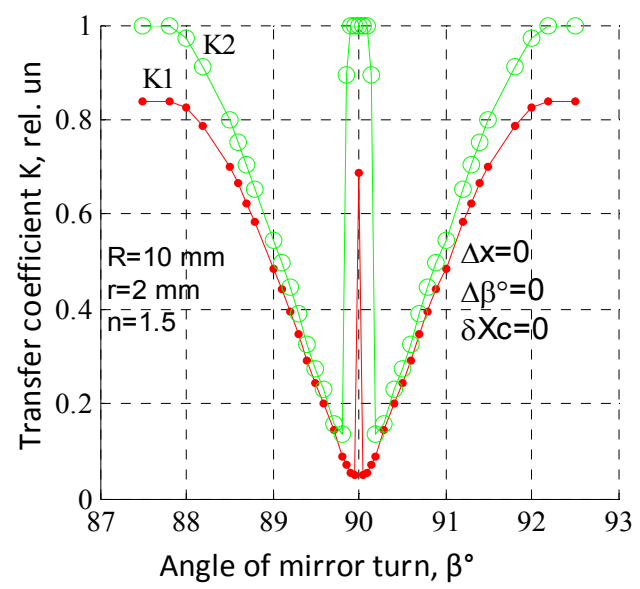

Fig. 11. Rotational dependences for the transfer coefficient in the ORC optical channel with a lens fiber-optic collimator, which are calculated in the model of facility with spherical focusing and collimating lenses for two types of light guides in the receiving channels. $\mathrm{K} 1$ is the relative transfer coefficient for ORC with single-mode light guides in the receiving channels; K2 - relative transfer coefficient for ORC with multimode light guides in the receiving channels.
The same results were obtained when calculating the construction with light beams of the diameters 1 and $2 \mathrm{~mm}$.

\subsection{Information capacity of ORC with the mirror compensator and fiber-optic collimators that provide wide optical beams}

As seen from Fig. 8, the amount of optical channels in ORC directly depends on the diameter of compensator optical sphere. The larger is the sphere diameter, the higher is the amount of channels that can be placed on its surface along the arch around the sphere. On the other hand, as calculations show, optical characteristics of the facility are worsened with increasing the compensator dimensions. I.e., the higher the amount of channels in the designed facility, the worse are optical characteristics of ORC for the set accuracy of manufacturing it.

For instance, if the diameters of holes for lenses in the inserts 16, 17 (Fig. 8) are assumed to be $1.5-$ $2.0 \mathrm{~mm}$, and the distance between holes is $0.5 \mathrm{~mm}$ (which is necessary to provide needed durability of construction), then the diameter of optical sphere $5 \mathrm{~mm}$ allows placing only three channels along the arc around the sphere (one - on the rotation axis, and two - on both sides of the axis under the angles $45^{\circ}$ ).

If the sphere diameter is increased up to $10 \mathrm{~mm}$, then there may be placed 5 optical channels. However, it considerably increases requirements to the accuracy of making the construction. For example, when the sphere diameter is $10 \mathrm{~mm}$, and with the same errors in the construction assemblage as in the construction with the 5 -mm optical sphere $\left(\Delta x=1 \mu \mathrm{m}, \delta X_{c}=5 \mu \mathrm{m} \quad\right.$ and $\Delta \beta=0.15^{\circ}$ ), optical losses will increase from 75 up to $90 \%$ in the variant of construction with collimating and focusing lenses of the diameter $1 \mathrm{~mm}$ and multimode light guides in optical channels of the facility.

Being based on the results of theoretical investigations [57], one can make the following conclusions. If the construction of movable transmitting mechanism contains tooth gears, then making the facility with the above mentioned accuracy is practically impossible. Especially, when it concerns the facility that designed to operate in harsh conditions of exploitation within a wide temperature range. Even for this practically unattainable accuracy, the values of optical losses and modulation distortions in the amplitude of optical signals during rotation of the rotor part remain too high.

\subsection{Optical characteristics of ORC with the mirror compensator and micro-lens optics}

Let us consider the variant of facility with application of micro-lenses on the butts of light guides.

Micro-optics allows realization of a narrow light beam with a small diameter at the output of single-mode light guide. In the case of narrow light beam, the optical rays after their splitting by the mirror pass near the optical axis of the system and are focused by the compensator optical sphere into the spot of small sizes (see Fig. 12). 

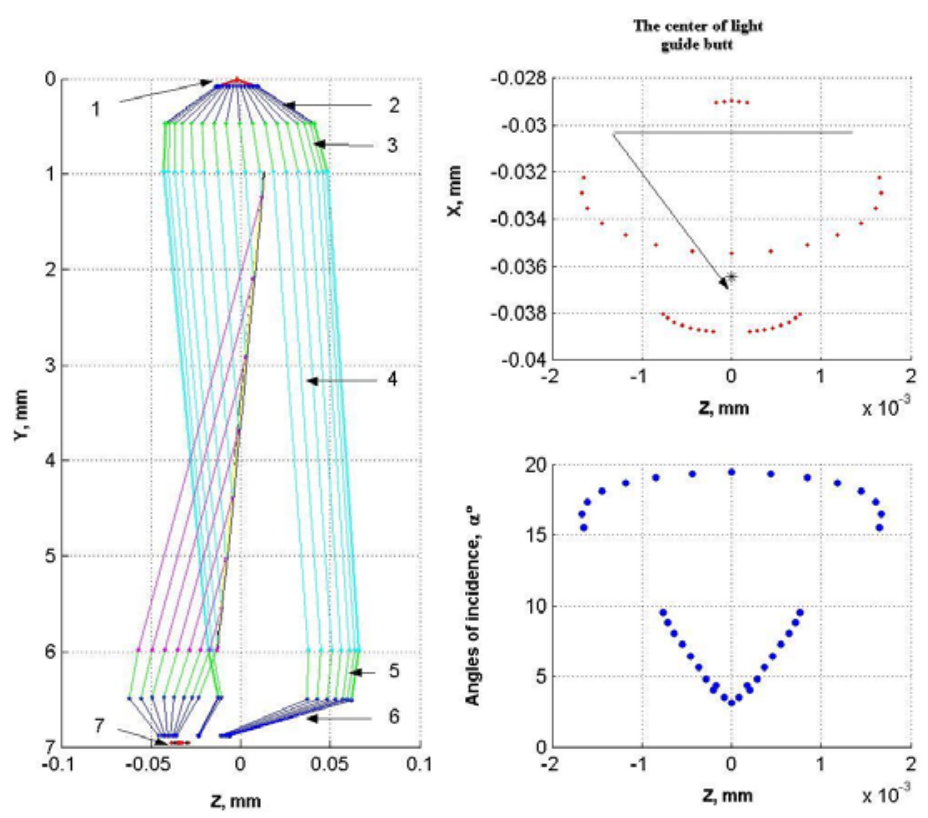

Fig. 12. Spatial distribution of rays in the ORC construction with microlenses for one of mirror positions: a) diagram of ray paths in ORC; b) distribution of ray coordinates within the plane of receiving light guide butt; c) distribution of ray angles of incidence onto the light guide butt. 1 and 7 - point light source and its image at the receiving light guide butt; 2 and 6 - ray paths in collimating and focusing lenses; 3 and 5 - ray paths between the optical sphere and microlenses; 4 - path of the part of rays that pass near the mirror.

Adduced in Fig. 12a is the calculated diagram of ray paths in the facility with the point source of light. Application of the point source of light instead of the long one allows revealing the typical features in behavior of this facility independently of source dimensions as well as saving the time for calculations.

Figs $12 \mathrm{~b}$ and $12 \mathrm{c}$ illustrate distributions for coordinates and angles of ray incidence onto the butt of receiving light guide for the construction with the following parameters: the radius of optical sphere $R=2.5 \mathrm{~mm}$, radius of the micro-lens $r=0.2 \mathrm{~mm}$, transversal shift of the point light source relatively to its nominal position $\Delta X=-2 \mu \mathrm{m}$, the maximum aperture angle of point source radiation is $7.5^{\circ}$.

As seen from Fig. 12a, in the case of micro-lenses all the rays pass near the optical axis (both those reflected from the mirror and those passing aside of the mirror) and, therefore, are focused into a spot of small sizes, the diameter of which is lower than $20 \mu \mathrm{m}$ (see Fig. 12b).

At the same time, despite sharp focusing the rays in this variant of construction one can also observe considerable losses and rotational oscillations in the optical signal value in ORC optical channels. In this case, the facility is exceedingly sensitive to the accuracy of making the construction and its assemblage. Even a small error in making the fiber-optic collimator results in the transverse shift of a point source relatively to the optical axis of collimating lens at the level of several micrometers, which increases optical losses and signal modulation up to the values higher than $90 \%$. The main reason for optical losses and additional modulation in this case is related with high angles of ray incidence onto the butt of receiving light guide after output from the short-focus lens when the rays exceed the aperture parameters of light guide.

As seen from Fig. 12c, when the transverse shift of the point light source reaches only $2 \mu \mathrm{m}$, a considerable part of rays at the output of short-focus lens falls onto the light guide butt under the angles 16 to 20 degrees, which essentially exceeds the operation aperture of fiber-optic cable.

The influence of the transverse shift $\Delta X$ of the point light source relatively to the center of the collimating micro-lens as well as radial beating $\delta X_{c}$ of the sphere on the transfer coefficient $K$ in ORC with multimode light guides $(\varnothing=50 \mu \mathrm{m}, \mathrm{NA}=0.19)$ at the receiving side of ORC are shown in Fig. 13a. The calculated curves for the dependence of the transfer coefficient on the value $\Delta \beta$ for angular beating of the mirror in the case of transverse shift of the point source $\Delta X=-3 \mu \mathrm{m}$ and radial beating of the sphere $\delta X_{c}= \pm 10 \mu \mathrm{m}$ is shown in Fig. 13b. The calculations were performed for the mirror angular position $\beta=89.8^{\circ}$.

As seen from the adduced figures, the radial shift of the point source by $3 \mu \mathrm{m}$ in the case of optical sphere radial beating $\delta X_{c} \pm 10 \mu \mathrm{m}$ leads to the value of optical losses at the level $50 \%$. The angular beating of the mirror with the value $0.3^{\circ}$ introduces additional optical losses and respective amplitude modulation of optical signals during mirror rotation from 50 up to $80 \%$.

Thus, being based on the considered dependences for the value of optical losses on the value of transverse shifting the point light source relatively to the microlens 


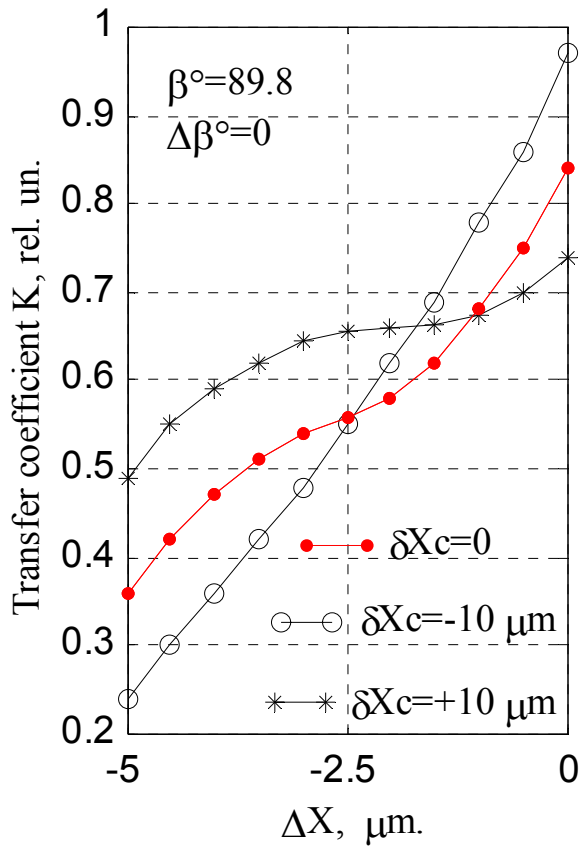

(a)

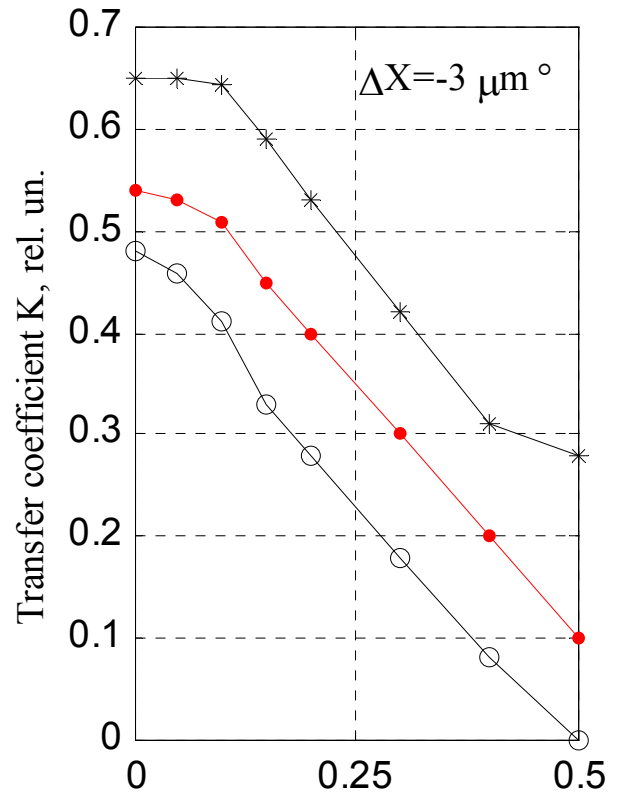

Mirror angular beating, $\Delta \beta^{\circ}$.

(b)

Fig. 13. Calculated dependences for the relative transmission coefficient in ORC with a point light source on the indexes of manufacturing accuracy.

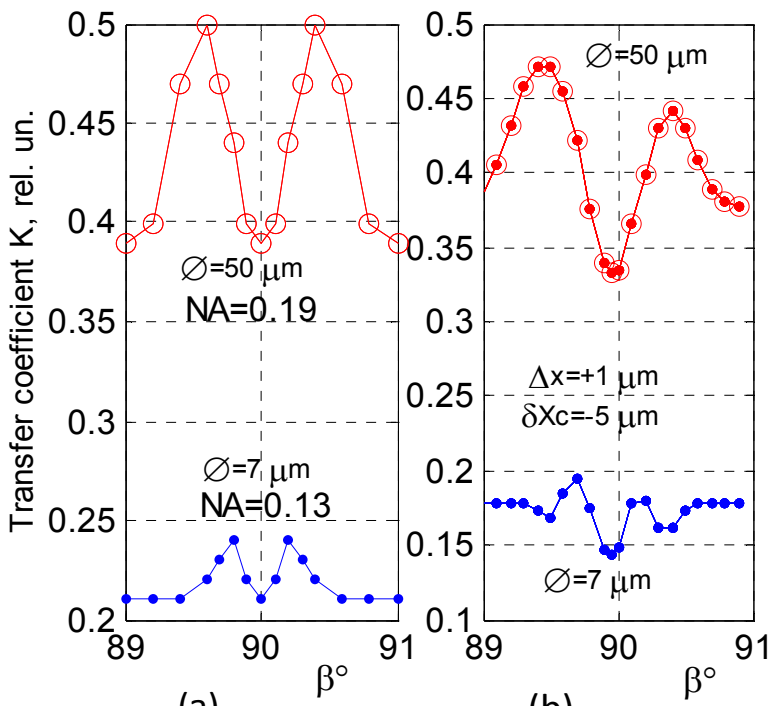

(b)

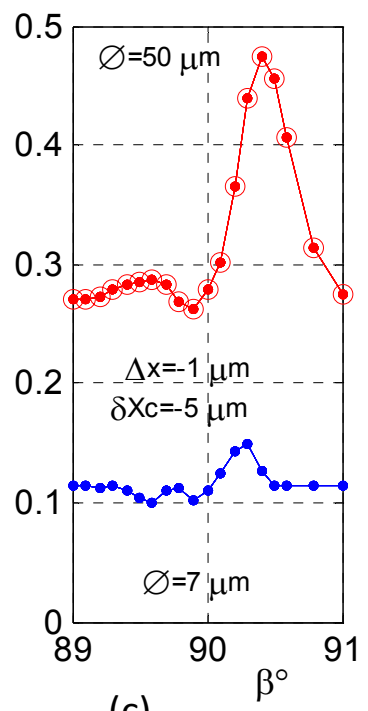

(c)

Fig. 14. Dependences of the signal transfer coefficient on the angle of mirror turn in ORC with microlenses of the diameter $0.4 \mathrm{~mm}$ and single-mode light guides in transmission channels as well as single-mode and multimode light guides in receiving channels: (a) for a geometrically ideal ORC; (b) for ORC with a light-guide butt shifted along the radius $(\Delta x=+1 \mu \mathrm{m}$, $\left.\delta X_{c}=-5 \mu \mathrm{m}\right)$; (c) for ORC with a light-guide butt shifted along the radius $\left(\Delta x=-1 \mu \mathrm{m}, \delta X_{c}=-5 \mu \mathrm{m}\right)$.

center, one can draw an important conclusion, namely: in optical channels of ORC of compensation operation with the mirror compensator and micro-optics, it seems reasonable to use optical sources only of small sizes that do not exceed $7 \mu \mathrm{m}$.

Depicted in Fig. 14 are the calculated characteristics for the transmission coefficient $K$ for ORC with the micro-lens of the diameter $0.4 \mathrm{~mm}$ and single-mode light guides having the diameter $\varnothing=7 \mu \mathrm{m}(\mathrm{NA}=0.13)$ (lower curves in the figure). Also adduced in this figure are the $K$ dependences on the angle of mirror turn in the case of using multimode light guides with the core diameter $50 \mu \mathrm{m}$ in the receiving channels, NA $=0.19$ (upper curves). 
Fig. 14a shows the characteristics for an ideal (from the geometrical viewpoint) facility. Depicted in Figs $14 \mathrm{~b}$ and $14 \mathrm{c}$ are the ORC characteristics typical for the optical sphere center shifted by the value $\delta X_{\mathrm{c}}=-5 \mu \mathrm{m}$ and the transverse shift of the transmitting light guide butt by $\Delta X= \pm 1 \mu \mathrm{m}$. These calculations were performed for the construction with parameters: $R=2.5 \mathrm{~mm}, r=0.2 \mathrm{~mm}, n=1.5$.

It is seen from Fig. 14a that in the geometrically ideal facility optical losses reach $80 \%$ of those in fiberoptic channels based on single-mode light guides, and $60 \%$ of those in optical channels where multimode light guides are used.

The latter variant could be considered as the acceptable one, but it is prevented by high sensitivity of facility performances to the construction assemblage. For instance, a small transverse shift of the transmitting light guide butt by $1 \mu \mathrm{m}$ reduces the transfer coefficient down to $10 \%$ in the optical channels with single-mode light guides, and does it down to $26 \%$ in those with multimode light guides at the receiving side (see Fig. 14c). In this case, modulation of the signal transfer coefficient during rotor rotation reaches the value $50 \%$ in the channel with the multimode light guide.

Thus, being based on the results obtained by using computer theoretical analysis of ORC construction known from the USA patent No 4447114 and containing the mirror compensator, one can draw the following conclusions:

1. ORC with mirror compensators can be capable only in the variant of using micro-optics, they are extremely sensitive to the accuracy of manufacturing the mechanism and optical components of the facility, a number of which should be made and mounted with the accuracy not worse than $1 \ldots 2 \mu \mathrm{m}$.

With this accuracy, the facility is low-technological and expensive, and its optical characteristics remain middling.

With increasing the diameter of compensator optical sphere, the ORC optical characteristics are worsened. So, the calculations indicate that, when the diameter of compensator optical sphere grows from 5 up to $14 \mathrm{~mm}$, optical losses increase from 80 up to $96 \%$ for the geometrically ideal construction with single-mode light guides, and from 60 up to $85 \%$ for the construction with multimode light guides.

2. Optical channels of the facility are onedirectional, since in the transmitting part only singlemode light guides with a small butt diameter can be applied, while in the receiving part multimode light guides with a large diameter of butt should be used.

3. The maximum amount of physical channels that can be really provided using this technical solution does not exceed 5-7 identities, since for larger amount of channels the requirements to ORC construction accuracy can be unreal.

4. The construction is not protected from mutual cross-interference between channels, which is caused by light scattering on optical inhomogeneities inside the sphere as well as on its surface.

\section{References}

1. Nickel H.U., Doleschel A. and Schmid M. Multichannel rotary joints for surveillance radars - State-of-the-art and future trends. 14-th Intern. Radar Symposium (IRS), Dresden, 2013, P. 282-287.

2. Glenn Dorsey, High-Speed Data Transmission and Rotary Platforms: Slip Rings, Fiber Optic Rotary Joints, and Multiplexers. MOOG Components Group. Application Note \#204. 2005. https:// www.researchgate.net/publication/242700826.

3. Ankerhold Georg, Laux Udo, Botter Oleg, Bestvater Marina Low-Loss Coupling of Rotating Single-Mode Fibers using Liquid Waveguides. SSD '09. 6-th Intern. Multi-Conf. on Systems, Signals and Devices, 2009.

4. Jia Da-Gong, Jing Wen-Cai, Zhang Yi-Mo, Zhou Ge, Zhang Jin, Tang Feng. Low-loss fiber optic rotary joint using C-lens collimators. Optoelectron. Lett. 2005. 1, Issue 3. P. 221-223.

5. W. Jing, D. Jia, F. Tang, H. Zhang, Y. Zhang, G. Zhou, J. Yu, F. Kong, and K. Liu, Design and implementation of a broadband optical rotary joint using C-lenses // Opt. Express, Vol. 12, No. 17, p. 4088-4093, Aug. 2004.

6. FORJ CATALOG 2009. Prinscetel Inc. http://pdf.directindustry.com/pdf/princetel/forjcatalog-2009o/29228-55142.html.

7. Dagong Jia, Wencai Jing, Yimo Zhang, Guanghui Wang, Feng Tang, Jin Zhang, Bidirectional dynamic data transmission through a rotary interface. Opt. Eng. 2005. 44, No. 5.

8. Introduction to Multiplexing in Fiber Optics. MOOG Components Group. Application Note \#213. http://www.moog.com/literature/MCG/ MultiplexingAppnote.pdf.

9. Farries M., Sturgess T. and Legg T. Transmission of digital and analogue data on a single mode fibre optic network for aircraft. Avionics, Fiber Optics and Photonics Technology Conference (AVFOP), 2012 IEEE, Cocoa Beach, FL, 2012. P. 72-73.

10. M. Oskar van Deventer, Fundamentals of Bidirectional Transmission over a Single Optical Fibre. Kluwer Academic Publishers, Dordrecht, The Netherlands, 1996.

11. Svechnikov G. and Shapar V. Optical rotary connector for transfer of data signals from fiber optic sensors placing on rotary objects. Specialty Fiber Optic Systems for Mobile Platforms. OE/FIBERS'91 SPIE Intern. Symposium, Boston, Massachusetts USA. Proc. SPIE. 1991. 1589. P. 24-31.

12. US Patent № 4,027,945, G02B 5/16. Optical sliprings. Myren L. Iverson. 07.06.77. 
13. UK Patent № 1587 531, G02B 5/16. Improvements relating to connectors. Andrew Foubister Aitkenhead, Melvyn Ramsay Bell, John Malcolm Morrison, Donald Ferguson Walker. 08.04.81.

14. USSR Inventing Certificate № 267390. S.V. Svechnikov, V.N. Shapar, R.P. Djangobegov, A.F. Ilyin et al. (USSR). Appl. No 3156922/3156923; filed. 08.12.1986; date of issue 04.01.1988, not published.

15. Japanese Patent № JPS63108310 (A), G02B6/26, G02B6/36. Optical Rotary Joint. Urata Harushige 13.05.1988.

16. European Patent Application № EP1134600 (A1), G02B6/36, G02B6/38, G02B6/40. Multiple path rotary optical connector. Woick Lothar, Loon Karel Henrik van. 19.09.2001.

17. Svechnikov G., Shapar V. Multichannel optical rotary connector for noncontact transfer of data signals between relatively moving bodies. OE/FIBERS'91, SPIE Intern. Symposium, Boston, Massachusetts, USA. Proc. SPIE. 1991. 1580. P. 391-394.

18. Shapar V., Svechnikov S. A small-size multichannel optical rotary joint with ring fiber optic converters. Kosmichna Nauka $i$ Tekhnologiya. SUPPLEMENT. 2003. 9, No. 2. P. 36-40 (in Ukrainian).

19. Shapar V.N. A small-sized multi-channel optical rotary joint for optical sensors based on rotating objects. The Seventh International Conference on Optoelectronics, Fiber Optics and Photonics, December 9-15, 2004, Cochin, India.

20. US Patent № 2012213472 (A1), G02B6/26. Fiber Bundle Based Passive Bi-Directional off Axis FORJ with Center Bore. Louis Violante, Boying B Zhang, Hong Zhang. 23.08.2012.

21. Johansson Mathias, Hard Sverker, Design, fabrication, and evaluation of a multichannel diffractive optic rotary joint. Appl. Opt. 1999. 38, No. 8. P. 1302-1310.

22. Shapar, V.M., Svechnikov, S.V., Indutnij I.Z. et al. A Multi-Channel Optical Rotary Joint on the Basis of Off-Axis Holographic Fresnel Lenses. 8-th Intern. Conf. on Laser and Fiber-Optical Networks Modeling, June 2006. P. 378-383.

23. US Patent № 4,854,662, G02B 6/36. Optical data signal apparatus for optically coupling plurality of data channels between stationary and rotating systems. Marvin F. Estes, Arnold W. Lungershausen. 08.08.1989.

24. US Patent № 4,875,756, G02B 6. Fresnel lens apparatus for optically coupling a plurality of data channels. Marvin F. Estes, Arnold W. Lungershausen. 24.10.1989.

25. UK Patent № 2183416, H04B 9/00. Apparatus for transmitting radiation between rotating members. Gordon Jeffrey Hanson, Martin Phillip Owen. 03.06.87.
26. US Patent № 4,519,670, G02B 5/14. Optical waveguide slip ring assembly. James G. Hamilton. 28.05.85.

27. DE Patent № 3,346,342 A1, H04B9/00. Optische Kopplungsvorrichtung mit Drehkupplung. E. Prinz, K. Schwepfinder, H. Bunke, H. Degwert. 12.06.1986.

28. US Patent № 4,109,997, G02B 23/02. Optical slip rings. Myren L. Iverson. 29.08.78.

29. Heizel T. and Martens G. Optical slip ring for offaxis high-bit-rate data transmission. Appl. Opt. 1986. 25, No. 5. P. 775-779.

30. US Patent № 4,934,783, G02B 6/26. Off-axis fiber optic rotary joint. Peter E. Jacobson. 19.06.1990.

31. DE Patent № 3326661 A1, G02B 5/176. Lichtwellenleiter-Drehkuppler. Weissenborn Bernd, Melchior Friedheim, Figge Johannes. 31.01.85.

32. US Patent № 4,525,025, G02B 5/14, G02B 7/26. Fiber optic rotary joint using a reflective surface and tangentially mounted rotor and stator optical fibers. Thomas C. Hohman, Norris E. Lewis, Michael B. Miller 25.06.1985.

33. CA Patent № 1239822 (A), IPC ${ }^{4}$ G02B6/36. Multiple Pass Optical Rotary Joint. Spenser William K., Oliver John B. 08.02.88.

34. Oliver John, Purdy John, Smith Graham, Data Integrity Key Design Factor in Fiber Optic Rotating Interfaces. SEA Technology. 1988. 29, No. 7. P. 32-36.

35. US Patent № 410,999, G02B 23/02. Optical slip rings. Myren L. Iverson. 29.08.78.

36. US Patent №4,872,737, G02B 6/36. Multi-port fiber optic rotary joint. Toshio Fukahori, Hideyuki Takashima, Hitoshi Morinaga. 10.10.89.

37. US Patent № 5,157,745, G02B 6/26. Multi-channel fiber optic rotary joint for single-mode fiber. Gregory H. Ames. 20.10.92

38. US Patent № 5,371,814, G02B 6/32. Passive, multi-channel fiber optic rotary joint assembly. Gregory H. Ames, Roger L. Morency. 06.12.94.

39. US Patent № 5,442,721, G02B 6/36. Fiber-optic rotary join with bundle collimator assemblies. Gregory H. Ames. 15.08.95.

40. US Patent № 6,301,405, G02B 6/26. Multi-channel fiber-optic rotary joint. Mithel J.Keil. 09.10.01.

41. International Patent № WO2008/077624 A1, G02B 6/36. Optical rotary joint with high return loss. Popp Gregor, Brau Josef, Rank Matthias. 03.07.08.

42. Jia Da-gong, Zhang Pei-Song, Jing Wen-Cai, Tan Jun, Zhang Hong-Xia, and Zhang Yi-Mo. Design of a multi-channel free space optical interconnection component. Optoelectron. Lett. 2008. 4 No. 6. P. 0407-0409.

43. US Patent № 2012,170888 (A1), G02B6/26. MultiChannel Electro-Magnetic Rotary Joint Using a Trapezoidal Metamaterial De-Rotating Mechanism. Violante Louis D, Zhang Boying B, Zhang Hong. 05.01.2012. 
44. US Patent № 4,447,114, G02B 7/26. Optical Slip Rings. Johan W. Koene. 30.08.83.

45. US Patent № 2006,133799 (A1), G02B5/08, G02B6/26, G02B6/42, H04B10/11, H04B10/25, H04B10/80. Contactless connector. Koitabashi Hiroyuki. 22.01.2006.

46. US Patent № 2006,202135 (A1), G02B27/00, H01F38/18, H04B10/11, H04B10/25. Non-Contact Connector. Koitabashi Hiroyuki. 14.10.2006.

47. US Patent № 2009,136175 (A1), G02B5/08, G02B6/26, G02B6/42, H04B10/11, H04B10/25, H04B10/80. Non-Contact Connector. Koitabashi Hiroyuki. 28.05.2009.

48. US Patent № 4,460,242, G02B 5/14, G02B 5/16. Optical Slip Ring. Ernest H. Birch. 17.07.1984.

49. US Patent № 4,943,137, G02B 6/32. Multichannel, off-axis, bi-directional fiber-optic slip ring. J. Alexander Speer. 24.07.90.

50. Machida H., Kobayashi H., Akedo J., Sawada K., Yasukawa T., Iino R. Optical rotary connector. Appl. Opt. 1988. 27, No.15. P. 3078-3080.

51. Shapar V., Svechnikov S., Oleksenko P. A multichannel optical rotary connector for space-based radar. Kosmichna Nauka $i$ Tekhnologiya. SUPPLEMENT. Vol. 9, \#2, p.30-35, 2003 (in Ukrainian).
52. Glenn F. Dorsey. Fiber Optic Rotary Joints - A Review. IEEE Transactions on Components, and Manufacturing Technology, V. CHTM-5, N.1, March 1982.

53. Churilovskyi V.N. Theory of Optical Devices. Moscow-Leningrad: Mashgiz, 1966. 564 p. (in Russian).

54. Smith W.J. Modern Optical Engineering. $3^{\text {rd }}$ edition. 2000.

55. Svechnikov S.V., Shapar V.N. Analysis of informative capacity of the optical rotary joint of compensative principle of action with the Dove prism and lens collimator. Measuring and computing devices in technological processes. 2010. 1. p. 48-55 (in Russian).

56. Sivulhin D.V. Basic Course of Physics. Optics. Moscow, Nauka, 1980. 752 p. (in Russian).

57. Shapar V.N. An analysis of rotary dependence of coefficient of transmission of optical rotary connector with mirror compensator. Measuring and computing devices in technological processes. 2007. 2. P. 48-53 (in Ukrainian).

58. Epshtein M.I. Measurements of Optical Radiation in Electronics. Moscow: Energoatomizdat, 1990. 254 p. (in Russian).

(To be continued). 